American Journal of Applied Sciences 5 (12): 1750-1753, 2008

ISSN 1546-9239

(C) 2008 Science Publications

\title{
A Note on the Bivariate Lagrange Interpolation Polynomials
}

\author{
Dan Bărbosu \\ North University of Baia Mare, Victoriei 76, 430122 Baia Mare, Romania
}

\begin{abstract}
The focus of the research is to obtain reccurence formulas for the bivariate Lagrange interpolation polynomials, similar to the reccurence formulas verified by the univariate Lagrange interpolation polynomials.
\end{abstract}

Key words: Bivariate (two dimensional) real valued function, divided differences, bivariate (two dimensional) divided differences, lagrange interpolation polynomials, bivariate lagrange interpolation polynomials

\section{PRELIMINARIES}

The bivariate Lagrange interpolation problem is very old and its classical solution is well known. Gordon, in 1969, in ${ }^{[5]}$, was the first who purposed a very nice and simple solution to this problem, based on the so called method of parametric extensions. It can be found also in ${ }^{[4,3,2]}$.

It is well known that the Lagrange univariate polynomial can be expressed in terms of univariate divided differences and many nice properties of above polynomials follow using the properties of the univariate divided differences ${ }^{[8,9,10,11]}$.

The notion of bivariate divided differences was introduced by Popoviciu ${ }^{[8]}$. These kind of differences were also considered by Ionescu ${ }^{[6]}$, in the particular case when the number of abscissas is equal with the number of coordinates. A general definition was gived by $\operatorname{Ivan}^{[7]}$.

Using the definition from ${ }^{[7]}$, in ${ }^{[2]}$ were investigated some properties of the bivariate divided differences. In the same paper $^{[2]}$, following the method of parametric extensions, the bivariate Lagrange interpolation polynomials were expressed in terms of bivariate divided differences and some of them approximation properties were recovered. Let us to recall some results from $^{[2]}$, which will be essentialy used in the present paper.

Suppose that $\mathrm{D}=[\mathrm{a}, \mathrm{b}] \times[\mathrm{c}, \mathrm{d}]$ is a bidimensional interval, $C(D)$ is the set of all real valued functions defined on $\mathrm{D}$ and $\mathrm{m}, \mathrm{n}$ are non-negative integers such that the points $\mathrm{x}_{0}, \ldots, \mathrm{x}_{\mathrm{m}} \in[\mathrm{a}, \mathrm{b}], \mathrm{y}_{0}, \ldots, \mathrm{y}_{\mathrm{n}} \in[\mathrm{c}, \mathrm{d}]$ are distinct.

The (m, n)-th order divided difference of $f \in C$ (D) with respect the knots $\left(x_{i}, y_{j}\right) \in D(i=0,1, \ldots m$, $\mathrm{j}=0,1, \ldots, \mathrm{n})$ is defined in ${ }^{[7]}$, by:

$$
\left[\begin{array}{l}
\mathrm{x}_{0}, \ldots, \mathrm{x}_{\mathrm{m}} \\
\mathrm{y}_{0}, \ldots, \mathrm{y}_{\mathrm{n}}
\end{array}\right]=\sum_{\mathrm{i}=0}^{\mathrm{m}} \sum_{\mathrm{j}=0}^{\mathrm{n}} \frac{\mathrm{f}\left(\mathrm{x}_{\mathrm{i}}, \mathrm{y}_{\mathrm{j}}\right)}{\mathrm{u}^{\prime}\left(\mathrm{x}_{\mathrm{i}}\right) \mathrm{v}^{\prime}\left(\mathrm{y}_{\mathrm{j}}\right)}
$$

where:

$$
\mathrm{u}(\mathrm{x})=\prod_{\mathrm{i}=0}^{\mathrm{m}}\left(\mathrm{x}-\mathrm{x}_{\mathrm{i}}\right), \quad \mathrm{v}(\mathrm{y})=\prod_{\mathrm{j}=0}^{\mathrm{n}}\left(\mathrm{y}-\mathrm{y}_{\mathrm{j}}\right)
$$

The bivariate Lagrange interpolation polynomial associated to the function $\mathrm{f} \in \mathrm{C}(\mathrm{D})$ and to the knots $\left(\mathrm{x}_{\mathrm{i}}\right.$, $\left.\mathrm{x}_{\mathrm{j}}\right) \quad(\mathrm{i}=0,1, \ldots, \mathrm{m}, \mathrm{j}=0,1, \ldots \mathrm{n})$ is the bivariate polynomial of minim degree wich interpolates the function $f$ on the knots $\left(x_{i}, x_{j}\right)$. It will be denoted by $\mathrm{L}_{\mathrm{m}, \mathrm{n}} \mathrm{f}\left(\begin{array}{c}\mathrm{x} ; \mathrm{x}_{0}, \ldots, \mathrm{x}_{\mathrm{m}} \\ \mathrm{y} ; \mathrm{y}_{0}, \ldots, \mathrm{y}_{\mathrm{n}}\end{array}\right)$ or, for more simplicity, by $\mathrm{L}_{\mathrm{m}, \mathrm{n}} \mathrm{f}(\mathrm{x}, \mathrm{y})$.

$\mathrm{In}^{\text {[2] }}$, using the method of parametric extensions, the polynomial $L_{m, n} f(x, y)$ was expressed in terms of bivariate divided differences under the form:

$$
\begin{aligned}
& L_{m, n} f(x, y)=f\left(x_{0}, y_{0}\right)+ \\
& +\sum_{i=1}^{m} \sum_{j=1}^{n}\left(x-x_{0}\right) \ldots\left(x-x_{i-1}\right)\left(y-y_{0}\right) . \\
& \cdots \cdot\left(y-y_{j-1}\right)\left[\begin{array}{l}
x_{0}, \ldots, x_{i} \\
y_{0}, \ldots, y_{j}
\end{array}\right] .
\end{aligned}
$$

The deal of the present paper is to establish a reccurence formula for the polynomials (3), which are similar to those verified by the univariate Lagrange polynomials.

\section{THE MAIN RESULT}

We start by recalling the following result: 
Theorem 1: Let $\mathrm{x}_{0}, \mathrm{x}_{1}, \ldots, \mathrm{x}_{\mathrm{m}} \in[\mathrm{a}, \mathrm{b}]$ be distinct points $\mathrm{f}$ $\in \mathrm{C}[\mathrm{a}, \mathrm{b}]$ and $\mathrm{L}_{\mathrm{m}} \mathrm{f}\left(\mathrm{x} ; \mathrm{x}_{0}, \ldots . \mathrm{x}_{\mathrm{m}}\right)$ is the Lagrange interpolation polynomial associated to $\mathrm{f}$ and to the knots $\mathrm{x}_{0}, \ldots . \mathrm{x}_{\mathrm{m}}$, the following equality ${ }^{[3,7,8,10,11]}$.

$$
\begin{aligned}
& \left(x_{m}-x_{0}\right) L_{m} f\left(x ; x_{0}, \ldots, x_{m}\right)= \\
& =\left(x-x_{0}\right) L_{m-1} f\left(x ; x_{1}, \ldots, x_{m}\right)- \\
& -\left(x-x_{m}\right) L_{m-1} f\left(x ; x_{0}, \ldots, x_{m-1}\right)
\end{aligned}
$$

holds.

The analogous of Theorem 1 for the bivariate Lagrange polynomials is expressed in.

Theorem 2: Let $\mathrm{x}_{0}, \ldots, \mathrm{x}_{\mathrm{m}} \in[\mathrm{a}, \mathrm{b}], \mathrm{y}_{0}, \ldots, \mathrm{y}_{\mathrm{n}} \in[\mathrm{c}, \mathrm{d}]$ be distinct points, $D=[a, b] \times[c, d], f \in C(D)$ and $\mathrm{L}_{\mathrm{m}, \mathrm{n}} \mathrm{f}\left(\begin{array}{l}\mathrm{x} ; \mathrm{x}_{0}, \ldots, \mathrm{x}_{\mathrm{m}} \\ \mathrm{y} ; \mathrm{y}_{0}, \ldots, \mathrm{y}_{\mathrm{n}}\end{array}\right)$ be the Lagrange bivariate interpolation polynomials associated to $\mathrm{f}$ and to the distinct knots $\left(\mathrm{x}_{\mathrm{i}}, \mathrm{y}_{\mathrm{j}}\right)(\mathrm{i}=0,1, \ldots \mathrm{m}, \mathrm{j}=0,1, \ldots, \mathrm{n})$. The following equality:

$$
\begin{aligned}
& \left(x_{m}-x_{0}\right)\left(y_{n}-y_{0}\right) L_{m, n} f\left(\begin{array}{l}
x ; x_{0}, \ldots, x_{m} \\
y ; y_{1}, \ldots, y_{n}
\end{array}\right)= \\
& =\left(x-x_{0}\right)\left(y-y_{0}\right) L_{m-1, n-1} f\left(\begin{array}{l}
x ; x_{1}, \ldots, x_{m} \\
y ; y_{1}, \ldots, y_{n}
\end{array}\right)- \\
& -\left(x-x_{0}\right)\left(y-y_{n}\right) L_{m-1, n-1} f\left(\begin{array}{l}
x ; x_{1}, \ldots, x_{m} \\
y ; y_{0}, \ldots, y_{n-1}
\end{array}\right)- \\
& -\left(x-x_{m}\right)\left(y-y_{0}\right) L_{m-1, n-1} f\left(\begin{array}{l}
x ; x_{0}, \ldots, x_{m-1} \\
y ; y_{1}, \ldots, y_{n}
\end{array}\right)+ \\
& +\left(x-x_{m}\right)\left(y-y_{n}\right) L_{m-1, n-1} f\left(\begin{array}{l}
x ; x_{0}, \ldots, x_{m-1} \\
y ; y_{0}, \ldots, y_{n-1}
\end{array}\right)
\end{aligned}
$$

holds.

Proof: Let $L_{m}^{x}, L_{n}^{y}$ be the parametric extensions of univariate Lagrange interpolation operator. According to (4) we have:

$$
\begin{aligned}
& \left(x_{m}-x_{0}\right) L_{m}^{x} f\left(x, y ; x_{0}, \ldots, x_{m}\right)= \\
& =\left(x-x_{0}\right) L_{m-1}^{x} f\left(x, y ; x_{1}, \ldots, x_{m}\right)- \\
& -\left(x-x_{m}\right) L_{m-1}^{x} f\left(x, y ; x_{0}, \ldots, x_{m-1}\right) \\
& \left(y_{n}-y_{0}\right) L_{n}^{y} f\left(x, y ; y_{0}, \ldots, y_{n}\right)= \\
& =\left(y-y_{0}\right) L_{n-1}^{y} f\left(x, y ; y_{1}, \ldots, y_{n}\right)- \\
& -\left(y-y_{n}\right) L_{n-1}^{y} f\left(x, y ; y_{0}, \ldots, y_{n-1}\right)
\end{aligned}
$$

Applying the operator $\mathrm{L}_{\mathrm{n}}^{\mathrm{y}}$ to the equation (6) it follows:

$$
\begin{aligned}
& \left(x_{m}-x_{0}\right) L_{n}^{y} L_{m}^{x} f\left(x, y ; x_{0}, \ldots, x_{m}\right)= \\
& =\left(x-x_{0}\right) L_{n}^{y} L_{m-1}^{x} f\left(x, y ; x_{1}, \ldots, x_{m}\right)- \\
& -\left(x-x_{m}\right) L_{n}^{y} L_{m-1}^{x} f\left(x, y ; x_{0}, \ldots, x_{m-1}\right) .
\end{aligned}
$$

But $L_{n}^{y} L_{m}^{x} f(x, y)=L_{m}^{x} L_{n}^{y} f(x, y)=L_{m, n} f(x, y)$, so that from (8) we get:

$$
\begin{aligned}
& \left(x_{m}-x_{0}\right) L_{m, n} f\left(\begin{array}{l}
x ; x_{0}, \ldots, x_{m} \\
y ; y_{0}, \ldots, y_{n}
\end{array}\right)= \\
& =\left(x-x_{0}\right) L_{m-1, n} f\left(\begin{array}{l}
x ; x_{1}, \ldots, x_{m} \\
y ; y_{0}, \ldots, y_{n}
\end{array}\right)- \\
& -\left(x-x_{m}\right) L_{m-1, n} f\left(\begin{array}{l}
x ; x_{0}, \ldots, x_{m-1} \\
y ; y_{0}, \ldots, y_{n}
\end{array}\right) .
\end{aligned}
$$

Multiplying (9) by $y_{n}-y_{0}$, it follows:

$$
\begin{aligned}
& \left(x_{m}-x_{0}\right)\left(y_{n}-y_{0}\right) L_{m, n} f\left(\begin{array}{l}
x ; x_{0}, \ldots, x_{m} \\
y ; y_{0}, \ldots, y_{n}
\end{array}\right)= \\
& =\left(x-x_{0}\right)\left(y_{n}-y_{0}\right) L_{m-1, n} f\left(\begin{array}{l}
x ; x_{1}, \ldots, x_{m} \\
y ; y_{0}, \ldots, y_{n}
\end{array}\right)- \\
& -\left(x-x_{m}\right)\left(y_{n}-y_{0}\right) L_{m-1, n} f\left(\begin{array}{l}
x ; x_{0}, \ldots, x_{m} \\
y ; y_{0}, \ldots, y_{n}
\end{array}\right) .
\end{aligned}
$$

According to (7), we have:

$$
\begin{aligned}
& \left(y_{n}-y_{0}\right) L_{m-1, n} f\left(\begin{array}{l}
x ; x_{1}, \ldots, x_{m} \\
y ; y_{0}, \ldots, y_{n}
\end{array}\right)= \\
& =\left(y-y_{0}\right) L_{m-1, n-1} f\left(\begin{array}{l}
x ; x_{1}, \ldots, x_{m} \\
y ; y_{1}, \ldots, y_{n}
\end{array}\right)- \\
& -\left(y-y_{n}\right) L_{m-1, n-1} f\left(\begin{array}{l}
x ; x_{1}, \ldots, x_{m} \\
y ; y_{0}, \ldots, y_{n-1}
\end{array}\right)
\end{aligned}
$$

and

$$
\begin{gathered}
\left(y_{n}-y_{0}\right) L_{m-1, n} f\left(\begin{array}{l}
x ; x_{1}, \ldots, x_{m} \\
y ; y_{0}, \ldots, y_{n}
\end{array}\right)= \\
=\left(y-y_{0}\right) L_{m-1, n-1} f\left(\begin{array}{l}
x ; x_{0}, \ldots, x_{m-1} \\
y ; y_{1}, \ldots, y_{n}
\end{array}\right)- \\
-\left(y-y_{n}\right) L_{m-1, n-1} f\left(\begin{array}{l}
x ; x_{0}, \ldots, x_{m-1} \\
y ; y_{0}, \ldots, y_{n-1}
\end{array}\right) .
\end{gathered}
$$


From (10), (11) and (12) one arrives to:

$$
\begin{aligned}
& \left(x_{m}-x_{0}\right)\left(y_{n}-y_{0}\right) L_{m, n} f\left(\begin{array}{l}
x ; x_{0}, \ldots, x_{m} \\
y ; y_{0}, \ldots, y_{n}
\end{array}\right)= \\
& =\left(x-x_{0}\right)\left(y-y_{0}\right) L_{m-1, n-1} f\left(\begin{array}{l}
x ; x_{1}, \ldots, x_{m} \\
y ; y_{1}, \ldots, y_{n}
\end{array}\right)- \\
& -\left(x-x_{0}\right)\left(y-y_{n}\right) L_{m-1, n-1} f\left(\begin{array}{l}
x ; x_{1}, \ldots, x_{m} \\
y ; y_{0}, \ldots, y_{n-1}
\end{array}\right)- \\
& -\left(x-x_{m}\right)\left(y-y_{0}\right) L_{m-1, n-1} f\left(\begin{array}{l}
x ; x_{0}, \ldots, x_{m-1} \\
y ; y_{1}, \ldots, y_{n}
\end{array}\right)+ \\
& +\left(x-x_{m}\right)\left(y-y_{n}\right) L_{m-1, n-1} f\left(\begin{array}{l}
x ; x_{0}, \ldots . x_{m-1} \\
y ; y_{0}, \ldots, y_{n-1}
\end{array}\right) .
\end{aligned}
$$

Remark 1: We shall verify the equality (5) in the following way. First, we observe that the left and respective right side of (5) are bivariate polynomials of degree at most $(m, n)$. Next, the left side of (5) verifies the equalities:

$$
\begin{aligned}
& \left(x_{m}-x_{0}\right)\left(y_{n}-y_{0}\right) L_{m, n} f\left(\begin{array}{l}
x_{i} ; x_{0}, \ldots, x_{m} \\
y_{j} ; y_{0}, \ldots, y_{n}
\end{array}\right)= \\
& =\left(x_{m}-x_{0}\right)\left(y_{n}-y_{0}\right) f\left(x_{i}, y_{j}\right), \\
& (\forall) i \in\{0,1, \ldots, m\},(\forall) j \in\{0,1, \ldots, n\} .
\end{aligned}
$$

Denoting by $\mathrm{Q}(\mathrm{x}, \mathrm{y})$ the right side of (5) we observe that:

$$
\begin{aligned}
& Q\left(x_{0}, y_{0}\right)= \\
& =\left(x_{0}-x_{m}\right)\left(y_{0}-y_{n}\right) L_{m-1, n-1} f\left(\begin{array}{l}
x_{0} ; x_{0}, \ldots, x_{m-1} \\
y_{0} ; y_{0}, \ldots, y_{n-1}
\end{array}\right)= \\
& =\left(x_{m}-x_{0}\right)\left(y_{n}-y_{0}\right) f\left(x_{0}, y_{0}\right), \\
& Q\left(x_{0}, y_{m}\right)= \\
& =\left(x_{0}-x_{m}\right)\left(y_{n}-y_{0}\right) L_{m-1, n-1} f\left(\begin{array}{l}
x_{0} ; x_{0}, \ldots, x_{m-1} \\
y_{n} ; y_{1}, \ldots, y_{n}
\end{array}\right)= \\
& =\left(x_{m}-x_{0}\right)\left(y_{n}-y_{0}\right) f\left(x_{0}, y_{n}\right), \\
& Q\left(x_{m}, y_{0}\right)= \\
& =\left(x_{m}-x_{0}\right)\left(y_{0}-y_{n}\right) L_{m-1, n-1} f\left(\begin{array}{l}
x_{m} ; x_{1}, \ldots, x_{m} \\
y_{0} ; y_{0}, \ldots, y_{n-1}
\end{array}\right)= \\
& =\left(x_{m}-x_{0}\right)\left(y_{n}-y_{0}\right) f\left(x_{m}, y_{0}\right), \\
& Q\left(x_{m}, y_{n}\right)= \\
& =\left(x_{m}-x_{0}\right)\left(y_{n}-y_{0}\right) L_{m-1, n-1} f\left(\begin{array}{l}
x_{m} ; x_{1}, \ldots, x_{m} \\
y_{n} ; y_{1}, \ldots, y_{n}
\end{array}\right)= \\
& =\left(x_{m}-x_{0}\right)\left(y_{n}-y_{0}\right) f\left(x_{m}, y_{n}\right) .
\end{aligned}
$$

For any $\mathrm{i} \in\{1,2, \ldots, \mathrm{m}-1\}$ and any $\mathrm{j} \in\{1,2, \ldots, \mathrm{n}$ 1) it follows:

$$
\begin{aligned}
& Q\left(x_{i}, y_{j}\right)= \\
& =\left(x_{i}-x_{0}\right)\left(y_{j}-y_{0}\right) f\left(x_{i}, y_{j}\right)-\left(x_{i}-x_{0}\right)\left(y_{j}-y_{n}\right) f\left(x_{i}, y_{j}\right)- \\
& -\left(x_{i}-x_{m}\right)\left(y_{j}-y_{0}\right) f\left(x_{i}, y_{j}\right)+\left(x_{i}-x_{m}\right)\left(y_{j}-y_{n}\right) f\left(x_{i}, y_{j}\right)= \\
& =\left(x_{i}-x_{0}\right)\left(y_{n}-y_{0}\right) f\left(x_{i}, y_{j}\right)+\left(x_{i}-x_{m}\right)\left(y_{0}-y_{n}\right) f\left(x_{i}, y_{j}\right)= \\
& =\left(y_{n}-y_{0}\right)\left(x_{m}-x_{0}\right) f\left(x_{i}, y_{j}\right) .
\end{aligned}
$$

We can conclude that the left and respectively the right side of (5) are bivariate polynomials of degree at most $(\mathrm{m}, \mathrm{n})$ which coincide in the points $\left(\mathrm{x}_{\mathrm{i}}, \mathrm{y}_{\mathrm{j}}\right)$ for $\mathrm{i}=$ $0,1, \ldots \mathrm{m}$ and $\mathrm{j}=0,1, \ldots \mathrm{n}$. It follows that (5) holds for any $(x, y) \in D$.

We shall apply the Theorem 2 for obtaining a recurence formula for divided bivariate differences.

Corollary 1: For any distinct points $\left(\mathrm{x}_{\mathrm{i}}, \mathrm{y}_{\mathrm{j}}\right) \in \mathrm{D}=[\mathrm{a}$, $\mathrm{b}] \times[\mathrm{c}, \mathrm{d}] \mathrm{i}=0,1, \ldots \mathrm{m}, \mathrm{j}=0,1, \ldots \mathrm{n}$, the following identity:

$$
\begin{aligned}
& \left(\mathrm{x}_{\mathrm{m}}-\mathrm{x}_{0}\right)\left(\mathrm{y}_{\mathrm{n}}-\mathrm{y}_{0}\right)\left[\begin{array}{l}
\left.\mathrm{x}_{0}, \ldots, \mathrm{x}_{\mathrm{m}} ; f\right]= \\
\mathrm{y}_{0}, \ldots, \mathrm{y}_{\mathrm{n}}
\end{array}\right]= \\
& =\left(\mathrm{x}-\mathrm{x}_{0}\right)\left(\mathrm{y}-\mathrm{y}_{0}\right)\left[\begin{array}{l}
\mathrm{x}_{1}, \ldots, \mathrm{x}_{\mathrm{m}} ; f \\
\mathrm{y}_{1}, \ldots, \mathrm{y}_{\mathrm{n}}
\end{array}\right]- \\
& -\left(\mathrm{x}-\mathrm{x}_{0}\right)\left(\mathrm{y}-\mathrm{y}_{\mathrm{n}}\right)\left[\begin{array}{l}
\mathrm{x}_{1}, \ldots, \mathrm{x}_{\mathrm{m}} ; f \\
\mathrm{y}_{0}, \ldots, \mathrm{y}_{\mathrm{n}-1}
\end{array}\right]- \\
& -\left(\mathrm{x}-\mathrm{x}_{\mathrm{m}}\right)\left(\mathrm{y}-\mathrm{y}_{0}\right)\left[\begin{array}{l}
\mathrm{x}_{0}, \ldots, \mathrm{x}_{\mathrm{m}-1} ; f \\
\mathrm{y}_{1}, \ldots, \mathrm{y}_{\mathrm{n}}
\end{array}\right]+ \\
& +\left(\mathrm{x}-\mathrm{x}_{\mathrm{m}}\right)\left(\mathrm{y}-\mathrm{y}_{\mathrm{n}}\right)\left[\begin{array}{l}
\mathrm{x}_{0}, \ldots, \mathrm{x}_{\mathrm{m}-1} ; f \\
\mathrm{y}_{0}, \ldots, \mathrm{y}_{\mathrm{n}-1}
\end{array}\right]
\end{aligned}
$$

holds.

Proof: It is immediately that the coefficient of $x^{m} y^{n}$ in the left side of (5) is:

$$
\left(x_{m}-x_{0}\right)\left(y_{n}-y_{0}\right)\left[\begin{array}{l}
x_{0}, \ldots, x_{m} \\
y_{0}, \ldots, y_{n}
\end{array}\right],
$$

while in the right side of the same identity the coefficient of $x^{m} y^{n}$ is:

$$
\begin{aligned}
& {\left[\begin{array}{l}
\mathrm{x}_{1}, \ldots, \mathrm{x}_{\mathrm{m}} \\
\mathrm{y}_{1}, \ldots, \mathrm{y}_{\mathrm{n}}
\end{array}\right]-\left[\begin{array}{c}
\mathrm{x}_{1}, \ldots, \mathrm{x}_{\mathrm{m}} ; \mathrm{f} \\
\mathrm{y}_{0}, \ldots, \mathrm{y}_{\mathrm{n}-1}
\end{array}\right]-} \\
& -\left[\begin{array}{c}
\mathrm{x}_{0}, \ldots, \mathrm{x}_{\mathrm{m}-1} ; \mathrm{f} \\
\mathrm{y}_{1}, \ldots, \mathrm{y}_{\mathrm{n}}
\end{array}\right]+\left[\begin{array}{l}
\mathrm{x}_{0}, \ldots, \mathrm{x}_{\mathrm{m}-1} ; \mathrm{f} \\
\mathrm{y}_{0}, \ldots, \mathrm{y}_{\mathrm{n}-1}
\end{array}\right] .
\end{aligned}
$$


Taking the identity (5) into account and using the above remarks on arrives to (13).

Remark 2: In Corollary 1 we refinded the reccurence formula for bivariate divided differences obtained $\mathrm{in}^{[2]}$, using the method of parametric extensions. So, we have another confirmation for the validity of mentioned result.

\section{ACKNOWLEDGEMENT}

At end of this little note, I want to thank to professor M. Ivan, from the Technical University of Cluj-Napoca, which offered me his excellent monograph $^{[7]}$, which was the most important source of inspiration for the note.

\section{REFERENCES}

1. Barbosu, D., 2002. Aproximarea functiilor de mai multe variabile prin sume booleene de operatori liniari de tip interpolator. Eds. Risoprint, ClujNapoca.

2. Bărbosu, D., 2008. Two dimensional divided differences revisited (to appear).
3. Coman, Gh., 1995. Analiza numerica. Eds. Libris, Cluj-Napoca.

4. Delvos, J., Schempp, W., 1989. Boolean methods in interpolation and approximation. Longman Scientific and Technical.

5. Gordon, W.J., 1969. Distributive lattices and the approximation of multivariate functions. In: Proc. Symp. Approximation with Emphasis on Spline Functions (Madison, Wisc.). Schoenberg, I.J. Ed., pp: 223-277.

6. Ionescu, D.V., 1978. Diferente divizate, Ed. Academiei R.S.R., București.

7. Ivan, M., 2004. Elements of Interpolation Theory. Mediamira Science Publisher Cluj-Napoca.

8. Popoviciu, T., 1934. Sur quelques proprietés des fonctions convexes d'une et de deux variables réelles. Mathematica, 1-85.

9. Raşa, I. and T. Vladislav, 1997. Analiza numerica. Elemente introductive. Ed. Tehnica, Bucuresti.

10. Stancu, D.D., 1977. Curs si culegere de probleme de analiza numerica, I, lito. Univ. "Babeş-Bolyai" Cluj-Napoca.

11. Stancu, D.D., Gh. Coman, O. Agratini and R. Trâmbițaş, 2001. Analiză numerică şi teoria aproximarii, Vol. 1. 